\title{
Some identities of Genocchi polynomials arising from Genocchi basis
}

Taekyun Kim ${ }^{1 *}$, Seog-Hoon Rim² ${ }^{2}$ Dmitry V Dolgy ${ }^{3}$ and Sang-Hun Lee ${ }^{4}$

"Correspondence: tkkim@kw.ac.kr 1 Department of Mathematics, Kwangwoon University, Seoul, 139-701, South Korea

Full list of author information is available at the end of the article

\section{Abstract}

In this paper, we give some interesting identities which are derived from the basis of Genocchi. From our methods which are treated in this paper, we can derive some new identities associated with Bernoulli and Euler polynomials.

MSC: $11 \mathrm{~B} 68 ; 11580$

\section{Introduction}

As is well known, the Genocchi polynomials are defined by the generating function to be

$$
\frac{2 t}{e^{t}+1} e^{x t}=e^{G(x) t}=\sum_{n=0}^{\infty} G_{n}(x) \frac{t^{n}}{n !} \quad(\text { see }[1-9])
$$

with the usual convention about replacing $G^{n}(x)$ by $G_{n}(x)$.

In the special case $x=0, G_{n}(0)=G_{n}$ are called the $n$th Genocchi numbers. From (1), we note that

$$
G_{0}=0, \quad G_{n}(1)+G_{n}=2 \delta_{n, 1} \quad(\text { see }[10-16]),
$$

where $\delta_{n, k}$ is the Kronecker symbol.

$$
G_{n}(x)=(G+x)^{n}=\sum_{l=0}^{n}\left(\begin{array}{l}
n \\
l
\end{array}\right) G_{l} x^{n-l} \quad(\text { see }[6-8,17]) .
$$

Thus, by (2) and (3), we see that

$$
\frac{d}{d x} G_{n}(x)=n G_{n-1}(x), \quad \operatorname{deg} G_{n}(x)=n-1 .
$$

The $n$th Bernoulli polynomials are also defined by the generating function to be

$$
\frac{t}{e^{t}-1} e^{x t}=e^{B(x) t}=\sum_{n=0}^{\infty} B_{n}(x) \frac{t^{n}}{n !} \quad(\text { see }[14-16])
$$

with the usual convention about replacing $B^{n}(x)$ by $B_{n}(x)$.

In the special case $x=0, B_{n}(0)=B_{n}$ are called the $n$th Bernoulli numbers. By (5), we get

$$
B_{0}=1, \quad B_{n}(1)-B_{n}=\delta_{1, n} \quad(\text { see }[8,9,17])
$$


and

$$
B_{n}(x)=\sum_{l=0}^{n}\left(\begin{array}{l}
n \\
l
\end{array}\right) B_{l} x^{n-l}=\sum_{l=0}^{n}\left(\begin{array}{l}
n \\
l
\end{array}\right) B_{n-l} x^{l}
$$

The Euler numbers are defined by

$$
E_{0}=1, \quad(E+1)^{n}+E_{n}=2 \delta_{0, n} .
$$

The Euler polynomials are defined by

$$
E_{n}(x)=(E+x)^{n}=\sum_{l=0}^{n}\left(\begin{array}{l}
n \\
l
\end{array}\right) E_{n-l} x^{l} \quad(\text { see }[7-13,17]) .
$$

Let $\mathbb{P}_{n}=\{p(x) \in \mathbb{Q}[x] \mid \operatorname{deg} p(x) \leq n\}$ be the $(n+1)$-dimensional vector space over $\mathbb{Q}$. Probably, $\left\{1, x, \ldots, x^{n}\right\}$ is the most natural basis for $\mathbb{P}_{n}$. But $\left\{G_{1}(x), G_{2}(x), \ldots, G_{n+1}(x)\right\}$ is also a good basis for the space $\mathbb{P}_{n}$ for our purpose of arithmetical applications of Genocchi polynomials. Let $p(x) \in \mathbb{P}_{n}$. Then $p(x)$ can be expressed by $p(x)=\sum_{1 \leq k \leq n+1} b_{k} G_{k}(x)$.

In this paper, we develop some new methods to obtain some new identities and properties of Genocchi polynomials which are derived from the Genocchi basis.

\section{Genocchi basis and some identities of Genocchi polynomials}

Let us take $p(x) \in \mathbb{P}_{n}$. Then $p(x)$ can be expressed as a $\mathbb{Q}$-linear combination of $G_{1}(x)$, $G_{2}(x), \ldots, G_{n+1}(x)$ as follows:

$$
p(x)=\sum_{1 \leq k \leq n+1} b_{k} G_{k}(x)=b_{1} G_{1}(x)+b_{2} G_{2}(x)+\cdots+b_{n+1} G_{n+1}(x) .
$$

Now, let us define the operator $\tilde{\triangle}$ by

$$
\tilde{\triangle} p(x)=p(x+1)+p(x) .
$$

Then, by (10) and (11), we set

$$
g(x)=\tilde{\triangle} p(x)=\sum_{1 \leq k \leq n+1} b_{k}\left(G_{k}(x+1)+G_{k}(x)\right) .
$$

From (1), we note that

$$
\sum_{n=0}^{\infty}\left\{G_{n}(x+1)+G_{n}(x)\right\} \frac{t^{n}}{n !}=\frac{2 t}{e^{t}+1} e^{(x+1) t}+\frac{2 t}{e^{t}+1} e^{x t}
$$

By (2), (3) and (13), we get

$$
\frac{G_{n+1}(x+1)+G_{n+1}(x)}{n+1}=2 x^{n} .
$$

From (12) and (14), we get

$$
g(x)=\tilde{\triangle} p(x)=2 \sum_{1 \leq k \leq n+1} k b_{k} x^{k-1}
$$


For $r \in \mathbb{N}$, let us take the $r$ th derivative of $g(x)$ in (15) as follows:

$$
g^{(r)}(x)=\frac{d^{r} g(x)}{d x^{r}}=2 \sum_{1 \leq k \leq n+1} k(k-1) \cdots(k-1-r+1) b_{k} x^{k-1-r} .
$$

Thus, by (16), we get

$$
g^{(r)}(0)=\left.\frac{d^{r} g(x)}{d x^{r}}\right|_{x=0}=2(r+1) ! b_{r+1}
$$

From (11) and (17), we have

$$
b_{r+1}=\frac{1}{2(r+1) !}\left\{p^{(r)}(1)+p^{(r)}(0)\right\}
$$

where $p^{(r)}(a)=\left.\frac{d^{r} g(x)}{d x^{r}}\right|_{x=a}$.

Therefore, by (10) and (18), we obtain the following theorem.

Theorem 1 For $n \in \mathbb{N}$, let $p(x) \in \mathbb{P}_{n}$ with $p(x)=\sum_{1 \leq k \leq n+1} b_{k} G_{k}(x)$.

Then we have

$$
b_{k}=\frac{1}{2 k !}\left(p^{(k-1)}(1)+p^{(k-1)}(0)\right) \text {. }
$$

Let us assume that $p(x)=B_{n}(x)$. Then by Theorem 1 , we get

$$
B_{n}(x)=\sum_{1 \leq k \leq n+1} b_{k} G_{k}(x)
$$

where

$$
b_{k}=\frac{1}{2 k !}\left\{p^{(k-1)}(1)+p^{(k-1)}(0)\right\}=\frac{1}{2 k !}(n)_{k-1}\left\{B_{n-k+1}(1)+B_{n-k+1}\right\} .
$$

From (6) and (20), we have

$$
b_{k}=\frac{1}{2(n+1)}\left(\begin{array}{c}
n+1 \\
k
\end{array}\right)\left\{\delta_{n, k}+2 B_{n-k+1}\right\} .
$$

By (19) and (21), we get

$$
\begin{aligned}
B_{n}(x) & =\frac{1}{n+1} \sum_{1 \leq k \leq n-1}\left(\begin{array}{c}
n+1 \\
k
\end{array}\right) B_{n-k+1} G_{k}(x)+\frac{1}{2}\left(1+2 B_{1}\right) G_{n}(x)+\frac{1}{2(n+1)} 2 G_{n+1}(x) \\
& =\frac{1}{n+1} \sum_{1 \leq k \leq n-1}\left(\begin{array}{c}
n+1 \\
k
\end{array}\right) B_{n-k+1} G_{k}(x)+\frac{1}{n+1} G_{n+1}(x) .
\end{aligned}
$$

Therefore, by (22), we obtain the following theorem.

Theorem 2 For $n \in \mathbb{N}$, we have

$$
B_{n}(x)=\frac{1}{n+1} \sum_{1 \leq k \leq n-1}\left(\begin{array}{c}
n+1 \\
k
\end{array}\right) B_{n-k+1} G_{k}(x)+\frac{1}{n+1} G_{n+1}(x) .
$$


In particular, if we take $p(x)=E_{n}(x) \in \mathbb{P}_{n}$, then we have

$$
E_{n}(x)=\sum_{1 \leq k \leq n+1} b_{k} G_{k}(x)
$$

where

$$
b_{k}=\frac{1}{2 k !}\left\{p^{(k-1)}(1)+p^{(k-1)}(0)\right\}=\frac{1}{2 k !}(n)_{k-1}\left\{E_{n-k+1}(1)+E_{n-k+1}\right\}
$$

By (8) and (24), we get

$$
\begin{aligned}
b_{k} & =\frac{1}{2(n+1)}\left(\begin{array}{c}
n+1 \\
k
\end{array}\right)\left\{2 \delta_{n-k+1,0}-E_{n-k+1}+E_{n-k+1}\right\} \\
& =\frac{1}{n+1}\left(\begin{array}{c}
n+1 \\
k
\end{array}\right) \delta_{n+1, k} .
\end{aligned}
$$

From (23) and (25), we have

$$
E_{n}(x)=\frac{1}{n+1} G_{n+1}(x)
$$

Let us take $p(x) \in \mathbb{P}_{n}$ with

$$
p(x)=\sum_{0 \leq k \leq n} B_{k}(x) B_{n-k}(x)
$$

Then we have

$$
\begin{aligned}
\frac{d p(x)}{d x} & =p^{(1)}(x)=\sum_{k=1}^{n} k B_{k-1}(x) B_{n-k}(x)+\sum_{k=0}^{n-1}(n-k) B_{k}(x) B_{n-k-1}(x) \\
& =(n+1) \sum_{k=1}^{n} B_{k-1}(x) B_{n-k}(x), \\
\frac{d^{2} p(x)}{d x^{2}} & =p^{(2)}(x)=(n+1) n \sum_{k=2}^{n} B_{k-2}(x) B_{n-k}(x) .
\end{aligned}
$$

Continuing this process, we get

$$
\begin{aligned}
\frac{d^{k} p(x)}{d x^{k}} & =p^{(k)}(x)=(n+1) n \cdots(n+1-k+1) \sum_{l=k}^{n} B_{l-k}(x) B_{n-l}(x) \\
& =\frac{(n+1) !}{(n+1-k) !} \sum_{l=k}^{n} B_{l-k}(x) B_{n-l}(x) .
\end{aligned}
$$

From (27), we have

$$
p^{(k-1)}(1)=\frac{(n+1) !}{(n+2-k) !} \sum_{l=k-1}^{n} B_{l+1-k}(1) B_{n-l}(1) .
$$


By (6), we get

$$
\begin{aligned}
B_{l+1-k}(1) B_{n-l}(1) & =\left(\delta_{l+1-k, 1}+B_{l+1-k}\right)\left(\delta_{n-l, 1}+B_{n-l}\right) \\
& =\left\{\delta_{k, n-1}+B_{n-k}+B_{n-k}+B_{l+1-k} B_{n-l}\right\} .
\end{aligned}
$$

From (28) and (29), we have

$$
p^{(k-1)}(1)=\frac{(n+1) !}{(n+2-k) !}\left\{\delta_{k, n-1}+2 B_{n-k}+\sum_{k-1 \leq l \leq n} B_{l+1-k} B_{n-l}\right\}
$$

By Theorem 1, $p(x)=\sum_{0 \leq k \leq n} B_{k}(x) B_{n-k}(x)$ can be expressed by

$$
p(x)=\sum_{1 \leq k \leq n+1} b_{k}(x) G_{k}(x)
$$

where

$$
\begin{aligned}
b_{k} & =\frac{1}{2 k !}\left\{p^{(k-1)}(1)+p^{(k-1)}(0)\right\} \\
& =\frac{(n+1) !}{2 k !(n+2-k) !}\left\{\delta_{k, n-1}+2 B_{n-k}+2 \sum_{l=k-1} B_{l+1-k} B_{n-l}\right\} .
\end{aligned}
$$

Thus, by (31) and (32), we get

$$
\begin{aligned}
p(x)= & \frac{n(n+1)}{12} G_{n-1}(x)+\sum_{1 \leq k \leq n+1} \frac{1}{k}\left(\begin{array}{l}
n+1 \\
k-1
\end{array}\right) B_{n-k} G_{k}(x) \\
& +\sum_{1 \leq k \leq n+1} \frac{1}{k}\left(\begin{array}{l}
n+1 \\
k-1
\end{array}\right) \sum_{l=k-1}^{n} B_{l+1-k} B_{n-l} G_{k}(x) .
\end{aligned}
$$

Therefore, by (31) and (33), we obtain the following theorem.

Theorem 3 For $n \in \mathbb{N}$, we have

$$
\begin{aligned}
\sum_{k=0}^{n} B_{k}(x) B_{n-k}(x)= & \frac{n(n+1)}{12} G_{n-1}(x)+\sum_{1 \leq k \leq n+1} \frac{1}{k}\left(\begin{array}{c}
n+1 \\
k-1
\end{array}\right) B_{n-k} G_{k}(x) \\
& +\sum_{1 \leq k \leq n+1}\left(\sum_{k-1 \leq l \leq n} \frac{1}{k}\left(\begin{array}{l}
n+1 \\
k-1
\end{array}\right) B_{l+1-k} B_{n-l}\right) G_{k}(x) .
\end{aligned}
$$

\section{Competing interests}

The authors declare that they have no competing interests.

Authors' contributions

All authors contributed equally to the manuscript and typed, read, and approved the final manuscript.

\section{Author details}

'Department of Mathematics, Kwangwoon University, Seoul, 139-701, South Korea. ${ }^{2}$ Department of Mathematics Education, Kyungpook National University, Daegu, 702-701, South Korea. ${ }^{3}$ Hanrimwon, Kwangwoon University, Seoul, 139-701, South Korea. ${ }^{4}$ Division of General Education, Kwangwoon University, Seoul, 139-701, South Korea. 


\section{Acknowledgements}

Dedicated to Professor Hari M Srivastava.

The authors would like to express their gratitude for the valuable comments and suggestions of referees. This research was supported by Kwangwoon University in 2013.

Received: 21 December 2012 Accepted: 13 January 2013 Published: 11 February 2013

\section{References}

1. Araci, S, Acikgöz, M, Jolany, H, Seo, JJ: A unified generating function of the $q$-Genocchi polynomials with their interpolation functions. Proc. Jangjeon Math. Soc. 15(2), 227-233 (2012)

2. Araci, S, Erdal, D, Seo, JJ: A study on the fermionic $p$-adic $q$-integral representation on $\mathbb{Z}_{p}$ associated with weighted $q$-Bernstein and $q$-Genocchi polynomials. Abstr. Appl. Anal. 2011, Article ID 649248 (2011)

3. Bayad, A, Kim, T: Identities for the Bernoulli, the Euler and the Genocchi numbers and polynomials. Adv. Stud. Contemp. Math. 20(2), 247-253 (2010)

4. Bayad, A: Modular properties of elliptic Bernoulli and Euler functions. Adv. Stud. Contemp. Math. 20(3), 389-401 (2010)

5. Cangul, IN, Kurt, V, Ozden, H, Simsek, Y: On the higher-order w-q-Genocchi numbers. Adv. Stud. Contemp. Math. 19(1), 39-57 (2009)

6. Ding, D, Yang, J: Some identities related to the Apostol-Euler and Apostol-Bernoulli polynomials. Adv. Stud. Contemp. Math. 20(1), 7-21 (2010)

7. Dolgy, DV, Kim, T, Lee, B, Ryoo, CS: On the q-analogue of Euler measure with weight. Adv. Stud. Contemp. Math. 21(4), 429-435 (2011)

8. Kim, DS, Lee, N, Na, J, Park, KH: Identities of symmetry for higher-order Euler polynomials in three variables (I). Adv. Stud. Contemp. Math. 22(1), 51-74 (2012)

9. Kim, T: Some identities for the Bernoulli, the Euler and the Genocchi numbers and polynomials. Adv. Stud. Contemp. Math. 20(1), 23-28 (2010)

10. Kim, T: On the multiple $q-G e n o c c h i$ and Euler numbers. Russ. J. Math. Phys. 15(4), 481-486 (2008)

11. Kim, T: Some identities on the $q$-Euler polynomials of higher order and $q$-Stirling numbers by the fermionic $p$-adic integral on $\mathbb{Z}_{p}$. Russ. J. Math. Phys. 16(4), 484-491 (2009)

12. Ozden, H, Cangul, IN, Simsek, Y: Remarks on $q$-Bernoulli numbers associated with Daehee numbers. Adv. Stud. Contemp. Math. 18(1), 41-48 (2009)

13. Rim, S-H, Jeong, J: On the modified $q$-Euler numbers of higher order with weight. Adv. Stud. Contemp. Math. 22(1), 93-98 (2012)

14. Ryoo, CS: Calculating zeros of the twisted Genocchi polynomials. Adv. Stud. Contemp. Math. 17(2), 147-159 (2008)

15. Simsek, Y: Generating functions of the twisted Bernoulli numbers and polynomials associated with their interpolation functions. Adv. Stud. Contemp. Math. 16(2), 251-278 (2008)

16. Simsek, Y: Theorems on twisted L-function and twisted Bernoulli numbers. Adv. Stud. Contemp. Math. 11(2), 205-218 (2005)

17. Kim, DS, Kim, T: Some identities of higher order Euler polynomials arising from Euler basis. Integral Transforms Spec. Funct. (2012). doi:10.1080/10652469.2012.754756

doi:10.1186/1029-242X-2013-43

Cite this article as: Kim et al.: Some identities of Genocchi polynomials arising from Genocchi basis. Journal of Inequalities and Applications 2013 2013:43.

\section{Submit your manuscript to a SpringerOpen ${ }^{\circ}$ journal and benefit from:}

- Convenient online submission

Rigorous peer review

- Immediate publication on acceptance

Open access: articles freely available online

High visibility within the field

- Retaining the copyright to your article 